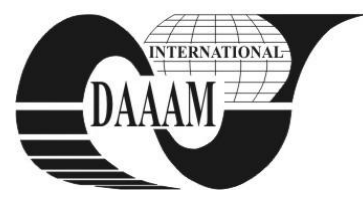

Annals of DAAAM for 2011 \& Proceedings of the 22nd International DAAAM Symposium, Volume 22, No. 1, ISSN 1726-9679 ISBN 978-3-901509-83-4, Editor B. Katalinic, Published by DAAAM International, Vienna, Austria, EU, 2011 Make Harmony between Technology and Nature, and Your Mind will Fly Free as a Bird

Annals \& Proceedings of DAAAM International 2011

\title{
FEM ANALYSIS OF PEDICLE SCREW-BONE INTERFACEFOR DIFFERENT INSERTION DIRECTIONS
}

\author{
POPESCU, D[iana] \& PARPALA, R[adu] C[onstantin]
}

\begin{abstract}
The paper presents a finite element analysis of three insertion directions of pedicle screw in lumbar vertebra, the purpose being to investigate the impact of the implantation trajectory over the stability of vertebral pedicle-screw interface, by comparing the maximal displacements obtained for the same pull-out force. Also, this information is helpful in the design process of a drilling template which can be used during surgery for increasing the pedicle screw positioning accuracy and its pull-out strength in the vertebra.
\end{abstract}

Key words: FEM, pedicle screw, lumbar vertebra, screw-bone interface

\section{INTRODUCTION}

Posterior spinal stabilizationis required for different types of pathologies and consists in using screws inserted in the pedicles and connected with rods for fusing the vertebrae. This surgical procedure imposes a precise technique for avoiding the damages of pedicles or neural structures.For this purpose, during the preoperative phase, the surgeon carefully analyses the bone quality, the pedicle morphology and orientation, and establishes the screw entry point in the vertebra (fig.1), as well as the screw type and dimensions (diameter and length).

In the clinical practice, the insertion trajectory of screws isusually along the pedicle axis for increasing the placement safety, but in the same time, the insertion direction must bechoosen to provide the strongest possible interface between bone and screw. In this context, there are severals ways for obtaining an enhanced interface between vertebral bone and pedicle screws: changing the design of the screw (Chatzistergos, et al., 2010), (Batulla, et al., 2006), (Patel et al., 2010) coating the screw surface for improving the contact or using reinforcing materials. Another possible solution, investigated in this paper with the use of finite element analysis (FEA) is to modify the screw implant trajectory for providing more contact with the cortical, rather than cancellous bone.This trajectory could be materialised during surgery by using a drilling template manufactured via a Rapid Prototyping process, customized for each pacient.

Analysis and characterisation of the interface between screws and bones represents an important research subject for several years due to the fact that clinical practice identifiesscrew loosening as a main cause of failure, despite a correct positioning of the implant. Therefore the literature presents biomechanical studies in which the pull-out strength is determined for different loads, insertion direction or fixation systems (Sterba et al., 2007), (Xu, et al., 2010), (Zhang, G.H., 2004), (Santoni, et al., 2008).However, to the best of the authors' knowledge no previous research has been done for establishing a finite element model of the interface between bone and pedicle screw inserted at different angles.

A review of finite elements(FE) studies for human spine is presented in (Jones\& Wilcox, 2008). The purpose of this research work is to establish a standard approach for verification and validation of different finite element models and materials properties used in different studies in the field for analysing bone-implant interface. Also, literature reports the use of FEM for improving the design and stability of screws, rods and screws-rods systems by elaborating and validating complete lumbar vertebra finite element models.

\section{METHOD}

A threedimensional model ofa L3 vertebra was generated in Mimics 10.01 from CT scan data (Van Sint, 1998). The model was exported in CATIA V5 and processed in different workbenches for obtaining a surface and then a solid model (fig.2). The vertebra was modelled as consisting in two parts: a cortical one at the exterior and a cancellous one in the interior.

The pedicle screw was modelled simplified, as a cylinder.

Three insertion trajectories were analyzedin ANSYS V12:1. along the pedicle axis; 2 . parallel with the spinous process; 3 . passing more medial-lateral ("closer" to the cortical bone)compared with direction 1 (fig.3). The deformation values for a pull-out force of $25 \mathrm{~N}$ were compared.

All the analysed trajectories were considered as starting from the same entry point, established at thelateral border of the superior facet where it intersects the midportion of the transverse process.

The material (considered isotropic and with linear elastic behaviour) properties for cortical and cancelous bone used in the FE model are presented in table 1, as mentioned in literature (Jones \& Wilcox, 2008).

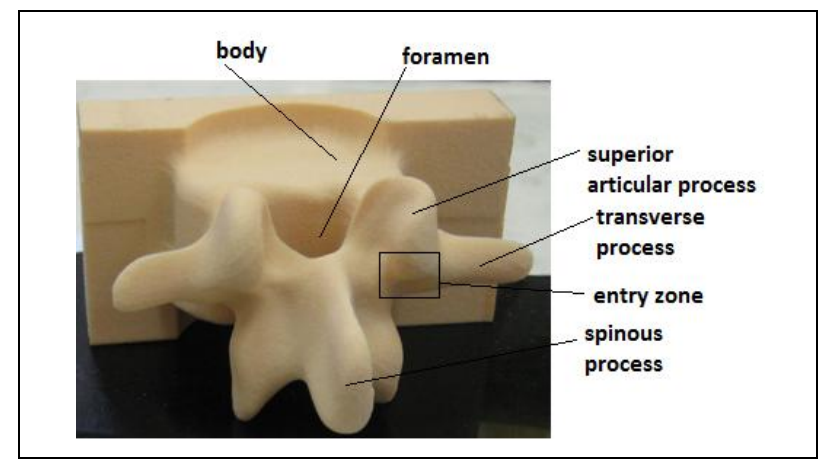

Fig. 1. Anatomy of a lumbar vertebra (L3)

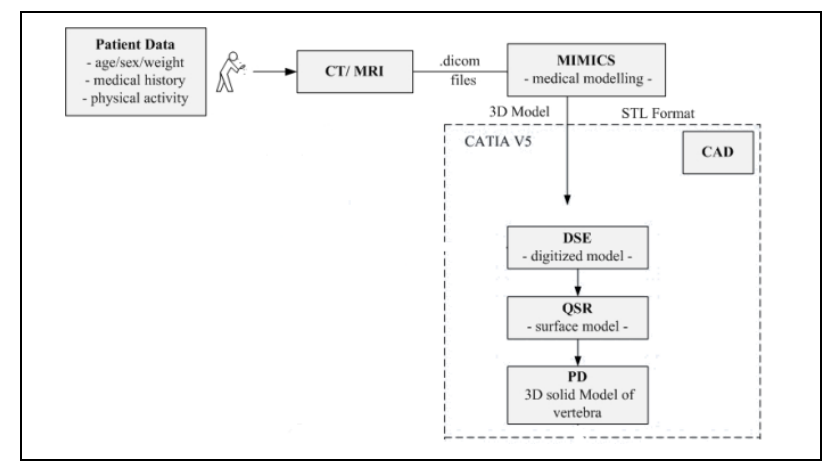

Fig.2. Mimics 10 - CATIA V5 integration 


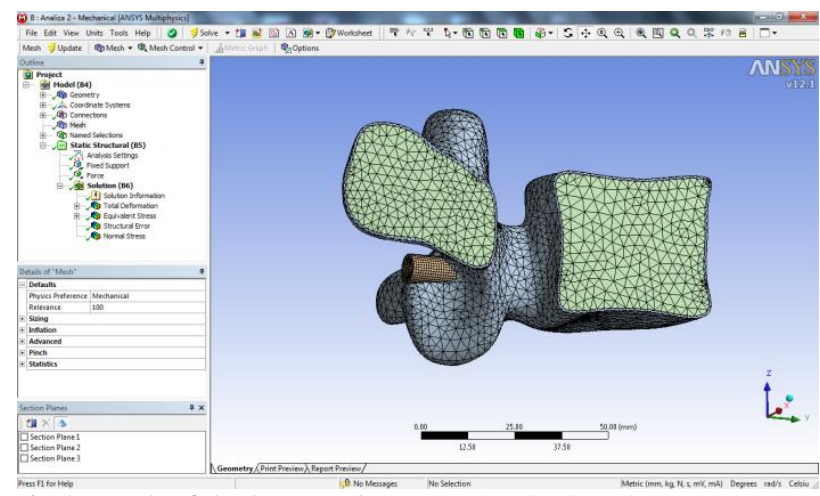

Fig.3. Mesh of the bone and screw - ANSYS V12

\begin{tabular}{|c|c|c|}
\hline & $\begin{array}{c}\text { Young Modulus } \\
(\mathrm{MPa})\end{array}$ & Poisson ratio \\
\hline Cortical bone & 780 & 0.3 \\
\hline Cancellous Bone & 100 & 0.2 \\
\hline Screw & 100000 & 0.3 \\
\hline Vertebral arch & 1000 & 0.45 \\
\hline Articular process & 1000 & 0.45 \\
\hline
\end{tabular}

Tab. 1. Material properties for bone and pedicle screw

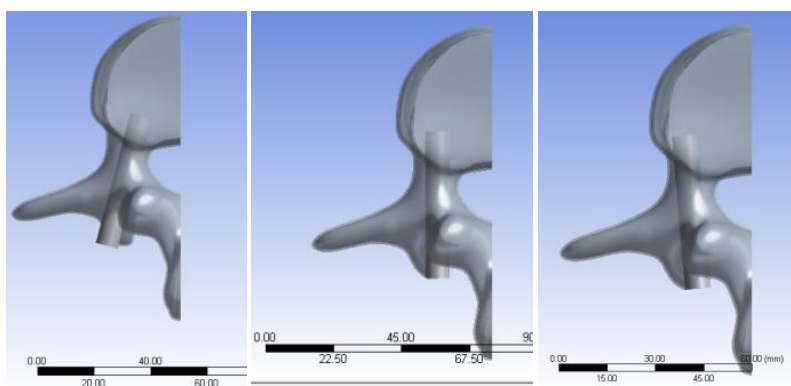

Fig. 4. Insertion trajectories for the pedicle screw

\section{RESULTS}

Analysing the total deformation and equivalent von Miss stressobtained in FEA (an example is presented in figure 3), the best insertion trajectory is parallel to the spinous process (table 2 ). These results are confirmed by the biomechanical tests presented in (Sterba et al., 2007) and (Santoni et al., 2008).

The explanation of this result is that the screw's thread, in this position, comes in contact with a larger part of the cortical bone - with better material properties compared to cancellous bone, this way improving the fixation strength. Due to the fact that this path is practically difficult to achieve, a drill guide, customized for each patient and manufactured using a Rapid Manufacturing process, could be a solution.

\section{DISCUSSION AND CONCLUSIONS}

The purpose of the research was to find a way to improve the longevity and stability of vertebral pedicle-screw interface. To the best of the authors' knowledge no previous research has been done for finite element modeling of the interface between bone and pedicle screw inserted at different angles, although biomechanical tests were performed in this sense.

The complexity of the problem resides in the difficulty to establish material properties or the correct boundary conditions considering the great variation between individuals. Therefore, further analysis will consider not only the use of a standard screw model, but also a better assessment of bone mechanical properties considering the new approach in which estimations of material properties are assigned based on the relation between Hounsfield units, bone mineral density (information available by quantitative computer tomography - QCT) and elastic modulus (Jovanovici, J.D., et al., 2010).

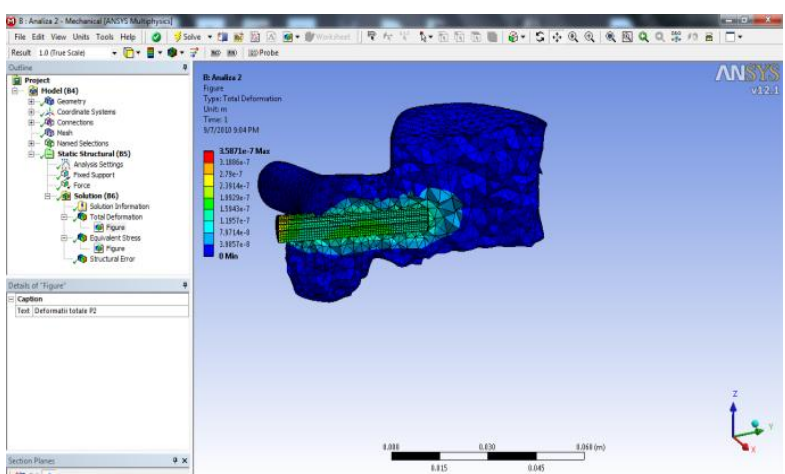

Fig. 5. Total deformation - trajectory 1

\begin{tabular}{|c|c|c|c|}
\hline & Trajectory 1 & Trajectory 2 & Trajectory 3 \\
\hline $\begin{array}{c}\text { Maximal } \\
\text { deformation }\end{array}$ & $3,5871 \cdot 10^{-7} \mathrm{~m}$ & $2,042 \cdot 10^{-7} \mathrm{~m}$ & $2,242 \cdot 10^{-7} \mathrm{~m}$ \\
\hline $\begin{array}{c}\text { Equivalent } \\
\text { von Miss } \\
\text { stress }\end{array}$ & $4,4411 \mathrm{MPa}$ & $3,362 \mathrm{MPa}$ & $3,3155 \mathrm{MPa}$ \\
\hline
\end{tabular}

Tab. 2. Results of FE analysis for three insertion trajectories

\section{ACKNOWLEDGEMENTS}

The work has been co-funded by the Sectoral Operational Programme Human Resources Development 2007-2013 of the Romanian Ministry of Labour, Family and Social Protection through the Financial Agreement POSDRU/89/1.5/S/62557.

\section{REFERENCES}

Battula, S., et al. (2006). Experimental evaluation of the holding power/stiffness of the self-tapping bone screws in normal and osteoporotic bone material. Clinical Biomechanics, 2:533-537

Chatzistergos, P.E., et al. (2010). A parametric study of cylindrical pedicle screw desgin implications on the pullout performance using an experimentally validated finiteelement model, Medical Engineering \& Physics, 32:145-154

Inceoglu, S., et al. (2007).Cortex of the pedicle of the vertebral arch. Part II: Microstructure, J Neurosurg Spine 7:347-351

Jones, A., Wilcox, R. (2008), Finite element analysis of the spine: Towards a framework of verification, validation and sensitivity analysis, Medical Engineering\&Physics, 30: 1287-1304

Jovanovici, J.D. et al. 2010. Finite element modeling of the vertebra with geometry and material properties retrieve from CT-scan data, FactaUniversitatis, Series: Mechanical Engineering. 8(1): $19-26$

Patel, P., et al. (2010). The effect of screw insertion angle and thread type on the pull-out strength of bone screws in normal and osteoporotic cancellous bone models, Medical Engineering \& Physics, 32(8):822-828

Santoni, B.G., et al. (2008). Cortical bone trajectory for lumbar pedicle screws, The Spine Journal, pp.1-8

Sterba, W., et al. (2007). Biomechanical analysis of different pedicle screw insertion angles, Clinical Biomechanics, 22:385-391

Van Sint J. S. (1998). Bone and Join CT-scan data, http://isbweb.org/data/vsj/index.html, The Laboratory of Human Anatomy and Embryology, Faculty of Medicine, University of Brussels

$\mathrm{Xu}$, H.G., et al. (2010), Finite element analysis of the screw in percutaneous axial lumbosacral interbody fusion, Orthopaedic Surgery, Vol.2, 3: 207-210

Zhang, G.H. (2004). Investigation of fixation screw pull-out strength on human spine, Journal of Biomechanics, 37:479485 